\title{
Complete and Early Vitrectomy for Endophthalmitis After Cataract Surgery: An Alternative Treatment Paradigm
}

This article was published in the following Dove Press journal:

Clinical Ophthalmology

\section{Bernard Dib (D) ${ }^{1-4}$ \\ Robert E Morris (iD) ${ }^{1-4}$ \\ Matthew H Oltmanns (iD ${ }^{1-4}$ \\ Mathew R Sapp ${ }^{1-4}$ \\ Jay $\mathrm{P}$ Glover ${ }^{5}$ \\ Ferenc Kuhn ${ }^{2,6,7}$ \\ 'Retina Specialists of Alabama, Birmingham, AL, USA; ${ }^{2}$ Helen Keller Foundation for Research and Education, Birmingham, AL, USA; ${ }^{3}$ University of Alabama at Birmingham (UAB), Department of Ophthalmology, Birmingham, AL, USA; ${ }^{4} \mathrm{UAB}$ Callahan Eye Hospital, Birmingham, AL, USA; ${ }^{5}$ Retina Consultants of Nashville, Nashville, TN, USA; ${ }^{6}$ Milos Eye Hospital, Belgrade, Serbia; ${ }^{7}$ Zagorskiego Eye Hospital, Krakow, Poland}

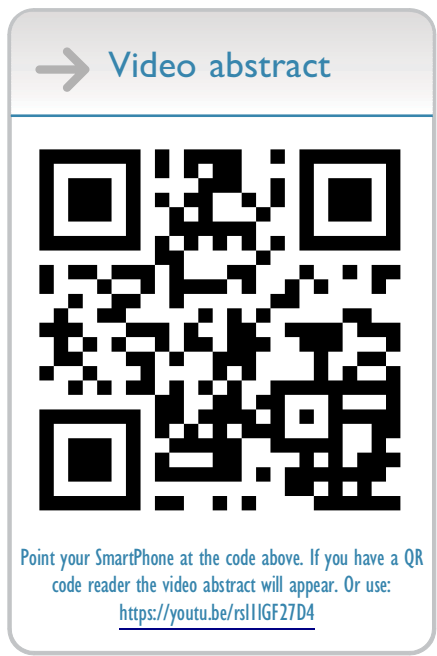

Correspondence: Robert E Morris Retina Specialists of Alabama, 2208 University Blvd, Ste. I0I, Birmingham, AL 35233, USA

Email rmorris@retinanetwork.com
Purpose: In this study, we report the treatment outcomes of complete and early vitrectomy for endophthalmitis (CEVE) after cataract surgery as the predominate initial treatment, accompanied by systemic antibiotics and retreatment of persistent or recurrent purulence (CEVE+).

Patients and Methods: Clinical features and microbiological factors were retrospectively reviewed in 62 eyes of 62 patients who were treated for acute postcataract endophthalmitis (APCE) occurring within three weeks of cataract surgery at Retina Specialists of Alabama, between 2007 and 2017.

Results: Visual acuity on presentation included light perception (LP) in 18 eyes (29\%) and hand motion (HM) in 23 eyes (37\%). Initial treatment was maximum possible vitrectomy in 48 eyes $(77 \%)$ and tap-and-inject in 14 eyes $(23 \%)$, with 38 eyes $(61 \%)$ receiving two or more treatments. Cultures for the first intervention were positive in 49 eyes $(79 \%)$ and virulent in 18 eyes (29\%). At a median follow-up time of five months, final visual acuity was $\geq 20 / 40$ in 49 eyes (79\%), between $20 / 50$ and 5/200 in seven eyes (11\%), and $<5 / 200$ in six eyes $(10 \%)$. Virulence was the strongest predictor of poor visual outcome. Retinal detachment occurred in four eyes $(6 \%)$, likely from necrotic retinal defects in each case.

Conclusion: Complete and early vitrectomy is a safe and effective initial treatment for APCE. When accompanied by systemic antibiotics and retreatment (CEVE+) of recurrent media opacification, it improves recovery of 20/40 or better visual acuity by approximately $50 \%$ compared to a predominantly tap-and-inject treatment paradigm. We recommend CEVE for fundus-obscuring APCE ( $75 \%$ of all cases) whenever the view is inadequate to rule out macular distress.

Keywords: endophthalmitis, postcataract endophthalmitis, vitrectomy, tap and inject, TAP, VIT, EVS, APCE

\section{Introduction}

With its ability to restore quality of life to those affected, cataract extraction is one of the most successful and impactful surgeries of modern medicine. As technology improves, surgeons and patients alike expect better outcomes. Despite these advancements, acute postcataract endophthalmitis (APCE) remains one of the most feared complications of cataract surgery. ${ }^{1,2}$

APCE causes significant morbidity, with almost half of patients losing reading visual acuity ( $\geq 20 / 40)$ and a quarter becoming legally blind (20/200 or worse) in the Endophthalmitis Vitrectomy Study (EVS) of $1995 .^{3}$ As the only prospective, 
randomized study for the treatment of APCE, the EVS established (core) vitrectomy (VIT) as equivalent to tapand-inject (TAP) for the $75 \%$ of eyes that presented with hand motion or better vision. The EVS also found no benefit from the use of intravenous antibiotics. These two findings encouraged office treatment of endophthalmitis with substantial cost savings and increased convenience. ${ }^{4}$

Today, vitrectomy has become small-gauge and sutureless, employing increasingly precise suction control and ultra-high-speed cutting rates of 10,000 cuts per minute (cpm) compared to the $600 \mathrm{cpm}$ available in the EVS era. Improved microscopes with panoramic viewing have also significantly enhanced the safety and efficiency of surgery. Despite these improvements, visual outcomes of APCE remain mostly unchanged or have even deteriorated since the EVS, with $\geq 20 / 40$ visual acuity achieved in half of all cases at best, in virtually all the studies that have reported on the treatment of APCE..$^{5-11}$

This situation is partly explained by the fact that the majority of APCE eyes are still treated with TAP based on the EVS recommendations and thus do not benefit from the extensive surgical advances made over the last two decades. In fact, some experts have questioned the applicability of the EVS to today's treatments. ${ }^{12-14}$ The main limitation of the EVS is that its vitrectomy was strictly a core VIT, with removal of (cortical vitreous) purulence on the retinal surface explicitly discouraged for fear of causing iatrogenic retinal tears. ${ }^{15}$ The primary goal of this partial vitrectomy was to "obtain specimens for culture and inject intravitreal antibiotics"; removal of infectious material was "a secondary goal."15

Today's instrumentation allows the performance of complete vitrectomy without compromising safety. ${ }^{16}$ The main advantage of a complete VIT over a core VIT is the removal of purulence in the cortical vitreous and on the retinal surface where most of the visually significant damage from endophthalmitis likely occurs. In fact, the major cause of vision loss in the EVS study was maculopathy, accounting for close to half of cases with impaired final visual acuity $(<20 / 40)$.

Therefore, in an effort to limit retinal injury caused by endophthalmitis (endophthalmitis retinopathy and endophthalmitis maculopathy), ${ }^{17}$ we prefer to perform Complete and Early Vitrectomy for Endophthalmitis $(\mathrm{CEVE})^{16}$ for all fundus-obscuring infections. TAP is reserved for early cases in which a view of the fundus is relatively preserved. Regardless of initial treatment, we follow patients closely with a low threshold to retreat significant deterioration of intraocular media clarity. ${ }^{18}$ CEVE-treated eyes with deteriorating clarity receive additional vitrectomy lavage and/or antibiotic injection $(\mathrm{CEVE}+)$. In this study, we describe the characteristics and outcomes of 62 APCE eyes treated with such an approach in the modern era of small gauge vitrectomy.

\section{Patients and Methods}

\section{Patients}

The study included a review of clinical features and microbial factors in all patients treated for APCE between 2007 and 2017 at Retina Specialists of Alabama (RSA), Birmingham. APCE was defined by the appearance of clinical symptoms and signs of endophthalmitis within three weeks of cataract surgery, regardless of whether or not cultures were ultimately positive. Cases that had cataract surgery combined with other ocular surgeries were excluded, as were cases that were deemed to be sterile postoperative inflammation. Since our goal was to assess the ability of predominantly CEVE treatment to restore good visual acuity (like the EVS), we excluded eyes with pre-existing maculopathy or ocular comorbidities limiting visual acuity to $<20 / 100$, and eyes with severe corneal opacification precluding even core VIT.

\section{Initial Treatment}

Patients with light perception (LP) presenting visual acuity were counseled that VIT was the preferred treatment. For hand motion (HM) or better visual acuity, patients were counseled that either TAP or VIT were reasonable options, but that we preferred VIT for fundus-obscuring endophthalmitis. Patients subsequently gave informed consent prior to all treatments.

As a result, CEVE was usually performed for anything but early endophthalmitis - defined by a preserved red reflex and a posterior view adequate to rule out retinal distress particularly in the macula (hemorrhages, vasculitis, retinal surface purulence). ${ }^{16,18}$ In such early APCE cases, TAP was typically recommended, and cultures were obtained by needle aspiration of the vitreous or anterior chamber. Vitrectomy was predominately 25 -gauge $(\mathrm{G})$ with occasional use of $23 \mathrm{G}$ or $27 \mathrm{G}$ vitrectomy. The primary goal of CEVE was to remove as much purulence as safely possible, particularly from the surface of the macula (macular hypopyon). ${ }^{17}$ If needed, CEVE included the creation of a posterior vitreous detachment and/or debridement of macular surface purulence (Supplementary Video 1). As the initial intravitreal treatment, $100 \%$ of patients received vancomycin $(1 \mathrm{mg}), 95 \%$ 
received ceftazidime $(2.25 \mathrm{mg}), 31 \%$ received dexamethasone $(0.4 \mathrm{mg})$, and only one patient received amikacin $(0.4 \mathrm{mg})$. At the conclusion of vitrectomy, $18 \%$ of eyes received $0.75 \mu \mathrm{g}$ of tPA intravitreal for fibrinolysis to aid removal of macular hypopyon (Supplementary Video 1).

Patients were admitted as inpatients (typically for 48-72h) in all cases of CEVE and in 9 of 14 TAP cases. Most patients received frequent topical treatment with fortified vancomycin, tobramycin, and prednisolone acetate while avoiding the supine position. Systemic moxifloxacin or vancomycin was given in most cases to achieve protective retinal and uveal tissue levels against grampositive bacteria ( $94 \%$ of all cultured bacteria in the EVS) aided by expected breakdown of the blood-ocular barrier. ${ }^{19}$ Inpatients were typically examined twice daily with the patient held NPO pending each examination result.

\section{Retreatment}

Both the decision to retreat and the form of retreatment were determined by the severity of retinopathy uncovered at the initial vitrectomy, gram stains and cultures, and the ability of an eye to maintain or improve the media clarity noted at presentation or established by the initial vitrectomy. Recurrent or increasing media opacity despite treatment was viewed as an indicator that inflammation and/or infection were inadequately controlled, and the eye was retreated accordingly with VIT or TAP. Antibiotics instilled during subsequent treatments were within safe doses $^{20}$ and tailored to available culture results and clinical suspicion.

\section{Visual Outcome Analysis}

Final visual acuities were measured using Snellen charts, with a minimum of three months follow-up required, unless visual acuity of $\geq 20 / 40$ was achieved earlier, or irreversible vision loss was deemed to have occurred. A Pearson's chisquare test with one degree of freedom was used to compare success rates in achieving $\geq 20 / 40$ visual acuity across different groups within this study, and between this study and the EVS.

\section{Results}

Sixty-nine eyes of 69 consecutive patients were identified that fulfilled the criteria for APCE as defined above. Similar to the EVS, a total of seven eyes were excluded: one based on severe corneal opacification precluding even core VIT (bacillus, with phthisical outcome); two due to lack of sufficient follow-up (both 20/100 at $<2$ weeks); and four based on a pre-existing visual acuity-limiting ocular comorbidity. The latter four excluded eyes achieved stable and clear status in the early postoperative period. Thus, 62 APCE patients remained and were analyzed in this series.

Table 1 summarizes the baseline characteristics and management features in this study as compared to the EVS. Visual acuity on presentation had a distribution that was similar to the EVS. Initial treatment was with as complete a VIT as possible in $77 \%$ of cases and with TAP in $23 \%$ of cases. Of the 62 eyes, $89 \%$ required at least one VIT (including 7 of 14 eyes initially treated with TAP), and $61 \%$ needed at least two treatments $(9 \%$ in the EVS).

First culture results (Table 2) were more likely to be positive in $\mathrm{LP} / \mathrm{HM}$ eyes (90\%) compared to counting fingers $(\mathrm{CF})$ or better eyes $(43 \%, \mathrm{p}=0.002)$. In 8 of 29 eyes $(28 \%)$ that were recultured at an average time of 1.8 days after initial treatment, the second culture results were positive. Presentation within two days of cataract surgery was a predictor of virulent growth (gram-negative bacteria, coagulase-positive Staphylococcus, and Streptococcus/ Enterococcus), with $50 \%$ of these eyes growing virulent bacteria compared to $24 \%(\mathrm{p}=0.07)$ of eyes that presented three or more days after cataract surgery. Still, $44 \%$ of all virulent infections presented five or more days after cataract surgery.

Table 3 presents an analysis of the final visual acuities of all eyes. Of the 62 eyes, 79\% achieved final visual acuity of $\geq 20 / 40$ ( $53 \%$ in the EVS, $p=0.0001)$. Those who presented early ( $<5$ days after cataract surgery) had better outcomes than those who presented later ( $\geq 5$ days), with $90 \%$ of the early group $(n=31)$ achieving $\geq 20 / 40$ visual acuity compared to $68 \%$ of the late group $(n=31, p$ $=0.29$ ). Although there was a similar prevalence of virulence in each group ( $32 \%$ vs $26 \%$, respectively), the patients in the late group were twice as likely to present with LP vision (39\% versus $19 \%$ ) and waited an average of 1.8 days between symptom onset and presentation as opposed to 0.5 days for the early group.

Profound vision loss $(<5 / 200)$ occurred in 26\% (5/19) of eyes with virulent growth; $16 \%$ (5/31) of late presenters $(\geq 5$ days); and $11 \%(2 / 18)$ of eyes with initial LP vision. Of the six eyes that suffered profound vision loss, five harbored virulent organisms, five presented late, while only two presented with LP vision. Virulence was hence the strongest predictor of profound visual loss, followed by delay in presentation and LP visual acuity on presentation. 
Table I Baseline Characteristics

\begin{tabular}{|c|c|c|c|}
\hline & & RSA (n=62) & EVS $(n=420)$ \\
\hline \multicolumn{2}{|l|}{ Median age (range) } & $73(34-96)$ & $75(24-95)$ \\
\hline \multicolumn{2}{|c|}{ Median days from CEIOL to presentation (range) } & $4(I-I 5)$ & $6(I-63)$ \\
\hline VA at presentation & $\begin{array}{l}\text { LP } \\
\mathrm{HM} \\
\mathrm{CF} \\
\geq 5 / 200\end{array}$ & $\begin{array}{l}18(29.0) \\
23(37.1) \\
10(16.1) \\
11(18.0)\end{array}$ & $\begin{array}{l}110(26.2) \\
185(44.1) \\
66(15.7) \\
59(14.1)\end{array}$ \\
\hline Initial treatment & $\begin{array}{l}\text { VIT } \\
\text { TAP }\end{array}$ & $\begin{array}{l}48(77.4) \\
14(22.6)\end{array}$ & $\begin{array}{l}218(51.9) \\
202(48.1)\end{array}$ \\
\hline Initial treatment with VIT by VA & $\begin{array}{l}\text { LP } \\
\mathrm{HM} \\
\mathrm{CF} \\
\geq 5 / 200\end{array}$ & $\begin{array}{l}17(94.4) \\
18(78.3) \\
9(90.0) \\
4(36.4)\end{array}$ & $\begin{array}{l}60(54.5) \\
99(53.5) \\
33(50) \\
26(44.1)\end{array}$ \\
\hline Number of treatments received & $\begin{array}{l}\geq 2 \\
\geq 3\end{array}$ & $\begin{array}{l}38(61.3) \\
14(22.6)\end{array}$ & $\begin{array}{l}39(9.3) \\
0(0)\end{array}$ \\
\hline Number of vitrectomies received & $\begin{array}{l}\geq 1 \\
\geq 2 \\
\geq 3\end{array}$ & $\begin{array}{l}55(88.7) \\
16(25.8) \\
3(4.8)\end{array}$ & $\begin{array}{l}230(54.8) \\
0(0) \\
0(0)\end{array}$ \\
\hline First culture & $\begin{array}{l}\text { Negative }^{\mathrm{a}} \\
\text { Positive } \\
\text { Gram-positive coagulase negative } \\
\text { Other gram-positive }^{\mathrm{b}} \\
\text { Gram-negative }^{\mathrm{c}} \\
\text { Polymicrobial }^{\text {Virulent }^{\mathrm{d}}}\end{array}$ & $\begin{array}{l}13(21.0) \\
49(79.0) \\
30(48.4) \\
17(27.4) \\
2(3.2) \\
0(0.0) \\
18(29.0)\end{array}$ & $\begin{array}{l}129(30.7) \\
291(69.3) \\
197(46.9) \\
65(15.5) \\
17(4.1) \\
12(2.9) \\
94(22.4)\end{array}$ \\
\hline Final VA follow-up time, months & $\begin{array}{l}\text { Mean } \\
\text { Median } \\
\text { Range }\end{array}$ & $\begin{array}{l}17.2 \\
5.8 \\
0.9-94\end{array}$ & $9-12$ \\
\hline
\end{tabular}

Notes: ${ }^{a}$ Includes equivocal cultures for EVS results. ${ }^{\mathrm{b}} 8$ Streptococcus, 6 Staphylococcus Aureus, I Propionibacterium, 2 Enterococcus. ${ }^{\mathrm{C} B}$ oth Pseudomonas. Includes all gram-negatives and other gram-positives (except Propionibacterium).

Abbreviations: RSA, Retina Specialists of Alabama; EVS, Endophthalmitis Vitrectomy Study; CEIOL, cataract extraction with intraocular lens implantation; VA, visual acuity; LP, light perception; HM, hand motion; CF, counting fingers; VIT, vitrectomy; TAP, tap-and-inject.

As outlined in Table 4 , vision loss $<20 / 40$ occurred in 13 eyes $(21 \%)$, and the main reason was maculopathy in six eyes $(10 \%)$, retinal detachment $(\mathrm{RD})$ in four eyes $(6 \%)$ and phthisis in three eyes (5\%). Maculopathy mainly included foveal atrophy and chronic macular edema, but also epimacular proliferation and one TAP-treated case of residual vitreomacular traction (VMT) for which the patient declined treatment, accepting 20/50 visual acuity.

All four cases of RD occurred in LP/HM eyes that underwent VIT, of which two were core VIT limited by keratopathy, and two were complete VIT that uncovered severe existent endophthalmitis retinopathy. All four cases of RD were thought to be secondary to necrotic defects, which were visualized in two cases. Three eyes $(5 \%)$ became phthisic with poor final visual acuity $<5 / 200$, all of which harbored virulent organisms.

\section{Discussion}

The EVS significantly enhanced our understanding of postcataract endophthalmitis and continues to provide clinicians with information that directly impacts clinical practice. Outcomes for APCE have not improved, however, and have arguably deteriorated over the last two decades, even as vitrectomy technology has rapidly advanced. In fact, all retrospective series published to date (except one limited report using CEVE) ${ }^{12}$ report a final visual acuity of $\geq 20 / 40$ in half of all cases at best $(53 \%$ overall in the EVS). ${ }^{3,5-11}$ 
Table 2 Microbiology Results

\begin{tabular}{|c|c|c|c|c|c|}
\hline \multicolumn{2}{|l|}{ First Culture } & $\begin{array}{l}\text { Negative } \\
3(21.0)\end{array}$ & $\begin{array}{l}\text { Nonvirulent } \\
31(50.0)\end{array}$ & $\begin{array}{l}\text { Virulent } \\
18(29.0)\end{array}$ & $\begin{array}{l}\text { Total } \\
62(100)\end{array}$ \\
\hline \multirow[t]{4}{*}{ VA at presentation } & LP & I (5.6) & $6(33.3)$ & II (6I.I) & 18 \\
\hline & HM & $3(13.0)$ & $17(74.0)$ & $3(13.0)$ & 23 \\
\hline & $\mathrm{CF}$ & $3(30.0)$ & $5(50.0)$ & $2(20.0)$ & 10 \\
\hline & $\geq 5 / 200$ & $6(54.5)$ & $3(27.3)$ & $2(18.2)$ & II \\
\hline \multirow[t]{2}{*}{ Initial treatment } & VIT & $3(6.2)$ & $29(60.4)$ & $16(33.3)$ & 48 \\
\hline & TAP & $10(71.4)$ & $2(14.3)$ & $2(14.3)$ & 14 \\
\hline \multirow[t]{3}{*}{ Days from CEIOL to presentation } & $\leq 2$ days & $4(33.3)$ & $2(16.7)$ & $6(50.0)$ & 12 \\
\hline & $\geq 3$ days & $9(18.0)$ & $29(58.0)$ & $12(24.0)$ & 50 \\
\hline & $\geq 5$ days & $5(16.0)$ & $18(58.0)$ & $8(26.0)$ & 31 \\
\hline Second culture & & $21(72.4)$ & $3(10.3)$ & 5 (I7.2) & $29(100)$ \\
\hline \multirow[t]{2}{*}{ Initial treatment } & VIT & $16(69.6)$ & $2(8.7)$ & $5(21.7)$ & 23 \\
\hline & TAP & $5(83.3)$ & I (I6.7) & $0(0)$ & 6 \\
\hline
\end{tabular}

Abbreviations: VA, visual acuity; LP, light perception; HM, hand motion; CF, counting fingers; VIT, vitrectomy; TAP, tap-and-inject; CEIOL, cataract extraction with intraocular lens implantation.

Enabled by the EVS recommendations, TAP remains the primary treatment for most APCE cases, particularly in the United States. This preference is evident in a recent survey, ${ }^{21}$ but also in several retrospective studies, ${ }^{5,6,8-10}$ three of which show VIT rates as low as $10 \%$ of all cases. Interestingly, this applied even to eyes presenting with LP vision, of which only $12 \%,{ }^{9} 16 \%,{ }^{6}$ and less than $50 \%{ }^{5}$ received a primary VIT despite the EVS recommendation for VIT treatment of all LP eyes. Thus, the trend towards TAP appears to have accelerated beyond the EVS guidelines, encouraged by the convenience and routine of office-based injection.

With complete VIT becoming considerably safer and less morbid than in the EVS era, a complete and early clean-up is now not only possible, but it also presents several potential advantages based on available experimental data. For instance, injecting rabbit eyes with bacterial culture fluid, even with the bacteria removed, resulted in substantially more severe and rapid toxicity than injecting live bacteria, extinguishing the electroretinogram within six hours. ${ }^{22}$ In another study, the inflammatory response induced by injecting live bacteria into rabbit eyes continued even after the bacteria reached an undetectable level within the eye. ${ }^{23}$ These experiments and others show that retinal damage is mostly due to toxin production and the host inflammatory response, and it can occur very rapidly. ${ }^{24,25}$ Therefore, early and thorough clearance of purulence and toxins is likely the most critical advantage of complete VIT over TAP, an advantage that was not fully realized by the EVS's explicitly partial VIT. $^{15}$

Enabled by technological improvements, the CEVE/ $\mathrm{CEVE}+$ paradigm improved visual outcomes, with $79 \%$ of eyes in this study achieving $\geq 20 / 40$ visual acuity compared to $53 \%$ in the EVS ( $\mathrm{p}=0.0001) ; 56 \%$ had VIT been used in all LP eyes in the EVS ( $\mathrm{p}=0.0001)$; and an aggregated average of $44 \%$ (range $25-52 \%$ ) in seven subsequent retrospective studies that mostly followed EVS guidelines. ${ }^{5-11}$

The CEVE/CEVE+ approach is intended to reduce the rate of maculopathy, the most common $(\sim 50 \%)$ cause of vision loss $<20 / 40$ in both the EVS and in this study. The term "endophthalmitis maculopathy" was introduced by Morris, Kuhn et al in $1995,{ }^{17}$ but OCT technology has improved our understanding of this concept, highlighting both reversible (edema, epimacular proliferation) and irreversible (atrophic) macular pathology long after endophthalmitis resolution. ${ }^{26}$ This is not surprising, as complete vitrectomy frequently reveals pus and inflammatory debris adhering to the surface of the macula (macular hypopyon, Supplementary Video 1). ${ }^{17}$ Further studies are needed to better characterize endophthalmitis maculopathy, both anatomically and functionally, with OCT, angiography, microperimetry, and electroretinography. Representative macular and retinal abnormalities noted in our patients are shown in Figures 1-3.

The rate of RD was $8 \%$ overall in the EVS and $6.4 \%$ in our study. Non-randomized studies both before ${ }^{27,28}$ and after ${ }^{29}$ the EVS have fostered speculation about the causative role of 
Table 3 Final Visual Acuity

\begin{tabular}{|c|c|c|c|c|c|}
\hline \multicolumn{2}{|l|}{ Total } & \multirow{2}{*}{$\begin{array}{l}\geq 20 / 40 \\
49(79.0)\end{array}$} & \multirow{2}{*}{$\frac{20 / 50-5 / 200}{7(1 / .3)}$} & \multirow{2}{*}{$\begin{array}{l}<5 / 200 \\
6(9.7)\end{array}$} & \multirow{2}{*}{$\begin{array}{l}\text { Total } \\
62(100)\end{array}$} \\
\hline & & & & & \\
\hline \multirow[t]{5}{*}{ VA at presentation } & LP & $10(55.5)$ & $6(33.3)$ & $2(I I . I)$ & 18 \\
\hline & $>\mathrm{LP}$ & $39(88.6)$ & I (2.3) & $4(9.1)$ & 44 \\
\hline & HM & $20(87.0)$ & I (4.3) & $2(8.7)$ & 23 \\
\hline & $\mathrm{CF}$ & $8(80.0)$ & $0(0.0)$ & $2(20.0)$ & 10 \\
\hline & $>5 / 200$ & II (100.0) & $0(0.0)$ & $0(0.0)$ & II \\
\hline \multirow[t]{2}{*}{ Initial treatment } & VIT & $36(75.0)$ & $6(12.5)$ & $6(12.5)$ & 48 \\
\hline & TAP & $13(92.9)$ & $\mathrm{I}(7 . \mathrm{I})$ & $0(0.0)$ & 14 \\
\hline \multirow[t]{2}{*}{ Organism } & Virulent & $10(52.6)$ & $4(21.0)$ & $5(26.3)$ & $19^{\mathrm{a}}$ \\
\hline & Negative or Non-Virulent & $39(90.7)$ & $3(7.0)$ & I (2.3) & 43 \\
\hline \multirow[t]{2}{*}{ Days from CEIOL to presentation } & $<5$ days & $28(90.3)$ & $2(6.4)$ & I (3.2) & 31 \\
\hline & $\geq 5$ days & $21(67.7)$ & $5(16.1)$ & $5(16.1)$ & 31 \\
\hline
\end{tabular}

Notes: ${ }^{a}$ Includes a patient that grew Staphylococcus coagulase-negative on first culture but Streptococcus on second culture.

Abbreviations: VA, visual acuity; LP, light perception; HM, hand motion; CF, counting fingers; VIT, vitrectomy; TAP, tap-and-inject; CEIOL, cataract extraction with intraocular lens implantation.

vitrectomy in endophthalmitis-associated RDs. Indeed, the EVS cited possible iatrogenic RD as its rational for limiting VIT. But this has no support in the EVS final data. In fact, $7.8 \%$ of EVS patients in the VIT group suffered an RD vs. $9 \%$ in the TAP group, a difference that was not significant $(p=0.66) .^{30}$

Importantly, the EVS found other significant associations with a higher rate of $\mathrm{RD}$, namely virulent growth (23\% RD rate in virulent gram-positives), LP presenting vision (16.4\% RD rate), and foregoing systemic antibiotics (11.2\% vs. $5.3 \% \mathrm{RD}$ rate). These associations indicate that endophthalmitis-associated RDs more likely occur as a result of necrotic retinal defects rather than iatrogenic tears. We have seen cases in which a completely necrotic, "moth-eaten" retina (Figure 4 and Supplementary Video 2) was found inferiorly in the areas where pus had settled by gravity. Complete VIT and adequate intraretinal antibiotic levels would logically tend to reduce necrotic defects secondary to such preretinal purulence.

All 13 eyes with final visual acuity $<20 / 40$ either harbored virulent organisms ( 9 eyes) and/or presented $>5$ days after cataract surgery (10 eyes). In all 12 of these eyes treated with initial vitrectomy, there was already either advanced endophthalmitis maculopathy/retinopathy uncovered ( 6 eyes) or keratopathy (usually corneal edema) precluding complete VIT (6 eyes). No eye treated with CEVE/CEVE+ failed to recover at least 20/40 vision if significant endophthalmitis maculopathy/retinopathy was not already present at the time of initial vitrectomy. This underscores the crucial role of the cataract surgeon in educating patients about the symptoms of endophthalmitis to avoid delayed presentations.

This encouraging outcome also highlights the importance of appropriate retreatment, which occurred in $61 \%$ of all patients at an average of 1.8 days after initial treatment, for both TAP and VIT retreatment. Eyes with substantial recurrent media opacification were retreated with complete VIT, while eyes with relatively maintained media clarity received TAP if infection control was still in doubt. Confirmation of the need to retreat inadequately responsive eyes is apparent from the fact that $27.5 \%$ of such recultured eyes in this study and $42 \%$ in the EVS remained culture positive at retreatment. ${ }^{31}$ Only $9 \%$ of eyes in the EVS had retreatment within one week of initial treatment.

Beyond initial toxin clearance, this represents a second significant advantage of primary CEVE: it restores clarity of the media, providing an extremely useful control parameter on which timely retreatment decisions can be based. In comparison, when TAP is performed on an eye with substantial media opacification, the decision to retreat is somewhat arbitrary since opacity and visual acuity do not typically improve within the next 24-48 hours, even if the infection is subsiding.

There are practical limitations to our approach. The CEVE + paradigm often entails two or more vitrectomies in the same week, including after hours. This situation requires a team approach, a reliable setup for emergent surgery, and ideally an inpatient facility, all resources that have become increasingly scarce in ophthalmology. Nevertheless, we hope that the 
Table 4 Reason for Vision Loss $(<20 / 40)$

\begin{tabular}{|c|c|c|c|c|c|c|}
\hline & D & Initial VA & Organism & Initial Treatment/Findings & Final VA & Main Reason \\
\hline \multirow[t]{9}{*}{ Virulent $(n=9)$} & 5 & LP & Strep & cVIT, severe maculopathy ${ }^{\mathrm{a}}$ & $20 / 60$ & Maculopathy \\
\hline & 5 & LP & Strep & cVIT, severe maculopathy ${ }^{\mathrm{a}}$ & $20 / 200$ & Maculopathy \\
\hline & 7 & LP & Staph Aureus & cVIT, severe maculopathy ${ }^{\mathrm{a}}$ & $\mathrm{CF}$ & Maculopathy \\
\hline & 2 & LP & Strep & iVIT due to keratopathy & $20 / 400$ & Maculopathy \\
\hline & 14 & LP & Strep & iVIT due to keratopathy & HM & Phthisis \\
\hline & 7 & $\mathrm{CF}$ & Pseudomonas & iVIT due to keratopathy & LP & Phthisis \\
\hline & 9 & $\mathrm{CF}$ & Staph Aureus & iVIT due to keratopathy & LP & Phthisis \\
\hline & 3 & LP & Staph Aureus & iVIT due to keratopathy & $20 / 400$ & RD \\
\hline & 2 & HM & Enterococcus & iVIT, severe retinopathy ${ }^{\mathrm{a}}$ & NLP & RD \\
\hline \multirow[t]{4}{*}{ Non-virulent or no growth $(n=4)$} & 7 & HM & NG & TAP & $20 / 50$ & Maculopathy \\
\hline & 6 & LP & Staph Coag - & cVIT, severe maculopathy ${ }^{\mathrm{a}}$ & $20 / 125$ & Maculopathy \\
\hline & 5 & HM & Staph Coag - & cVIT, severe retinopathy ${ }^{\mathrm{a}}$ & HM & RD \\
\hline & 5 & LP & Staph Coag - & iVIT due to keratopathy & $20 / 160$ & RD \\
\hline
\end{tabular}

Notes: ${ }^{\text {a}}$ Retinal hemorrhages, vasculitis, and pus accumulation on the surface of the retina

Abbreviations: D, days from cataract surgery to presentation; VA, visual acuity; CF, counting fingers; HM, hand motion; LP, light perception; NLP, no light perception; NG, no growth; cVIT, complete vitrectomy; iVIT, incomplete vitrectomy; TAP, tap-and-inject; RD, retinal detachment.

improved visual outcomes presented in this report will catalyze additional investigations and attainable practice pattern adjustments in treating this iatrogenic and frequently devastating disease.

Although our presenting patient cohort was similar to that of the EVS, the size, retrospective nature, and differing pharmacologics used in this report limit its comparability to the EVS and our ability to attribute the very substantial visual acuity improvements relative to the EVS solely to the use of CEVE/CEVE+. Nevertheless, we believe preferential use of CEVE (77\%), frequent retreatment $(61 \%)$, and use of appropriate systemic antibiotics



Figure I Extensive chorioretinal damage from Enterococcus Faecalis endophthalmitis. (A) Extensive hemorrhagic retinopathy noted during vitrectomy performed one day after cataract surgery. (B) Fundus photos six weeks postoperatively shows persistence of hemorrhages encircling the posterior pole. (C) Fundus photos six months postoperatively shows resolution of hemorrhages, severe chorioretinal atrophy, and attenuation of retinal blood vessels. (D) OCT one year postoperatively shows severe macular atrophy with a central foveal thickness of 183 microns. Final visual acuity is counting fingers. 


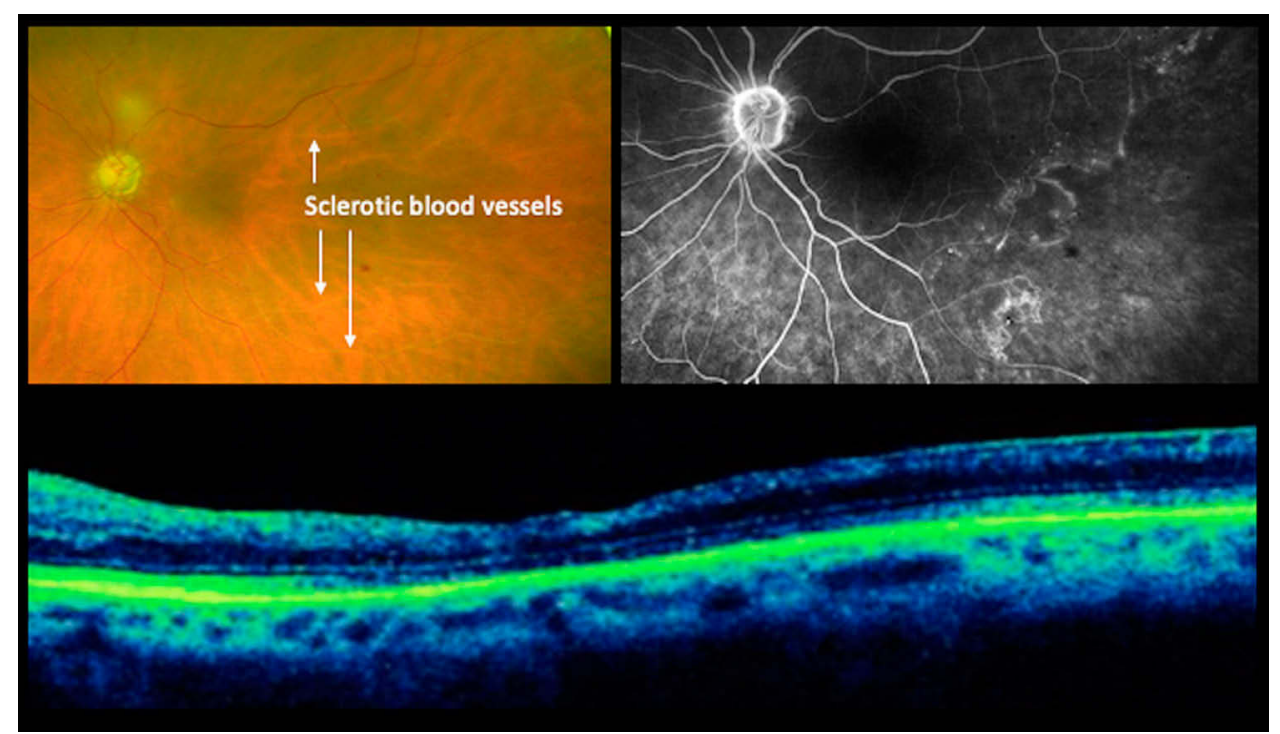

Figure 2 Permanent endophthalmitis retinopathy after coagulase-negative Staphylococcus endophthalmitis. (A) Fundus appearance shows sclerotic arterioles temporally. (B) Fluorescein angiography reveals extensive nonperfusion temporally and enlarged FAZ. (C) OCT at three years demonstrates severe macular atrophy. Final visual acuity is $20 / / 25$.

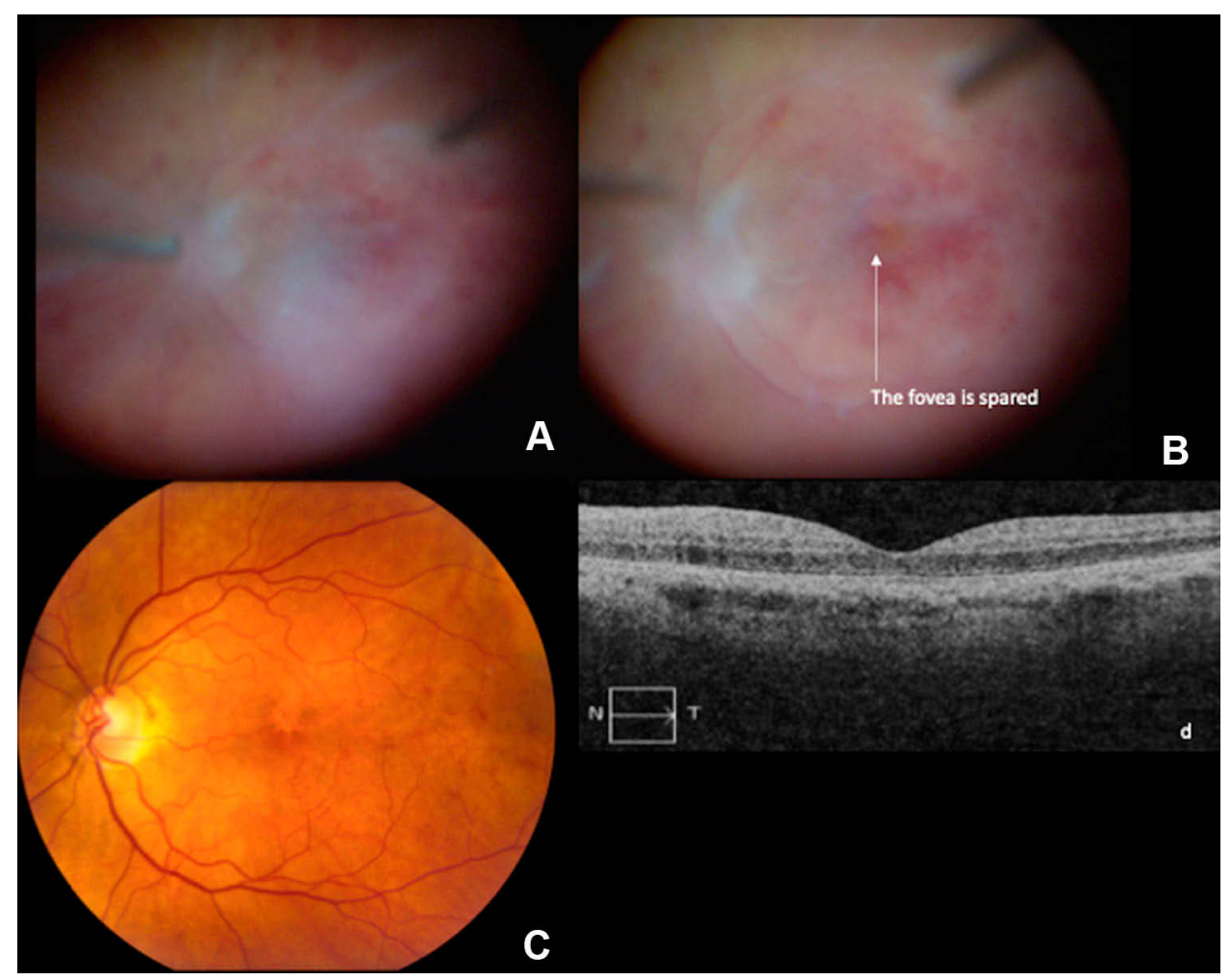

Figure 3 Coagulase-negative Staphylococcus with severe maculopathy sparing the fovea. (A) Intraoperative image of macular hypopyon with temporal macular hemorrhages, and an obscured fovea. (B) After removal of the macular hypopyon, the fovea is visible and is spared of hemorrhage. (C) Fundus three weeks postoperatively shows juxtafoveal atrophy of the retinal pigment epithelium. (D) Postoperative OCT reveals a normal fovea with preserved photoreceptors.

Notes: The external limiting membrane/ellipsoid disruption paracentrally in areas of previous hemorrhage. Final visual acuity is 20/25.

(to achieve intraretinal levels protective against bacteria at the vitreoretinal interface) account for the majority of the observed improvement.
Based on these encouraging results and the substantial advances seen in vitreoretinal surgery since the EVS, we are currently planning a prospective clinical trial of CEVE/ 


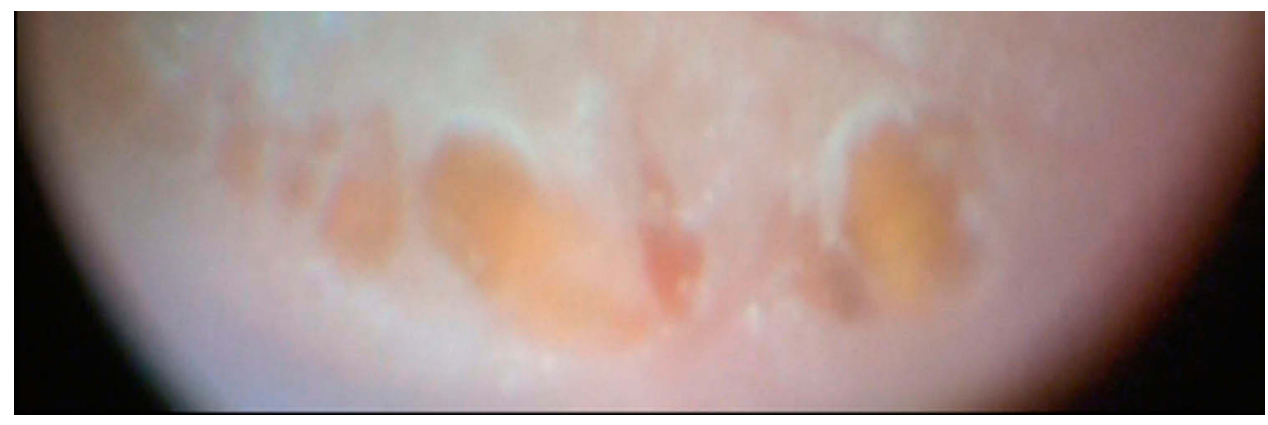

Figure 4 Retinal detachment resulting from large necrotic holes seen inferiorly two weeks after resolved Streptococcal endophthalmitis. This is sometimes seen inferiorly where preretinal pus settles by gravity in the upright position (ocular hypopyon). See Video 2 for more details.

CEVE+ that will allow a closer comparison to the EVS and its conclusions that remain widely followed standards of care.

\section{Conclusion}

This study is the first detailed report of improved visual outcomes for APCE since the EVS 25 years ago. Akin to treating infections elsewhere in the body with abscess drainage, ${ }^{32}$ recurrent lavage, and sustained antibiotic dosing, we believe CEVE/CEVE+ rapidly restores and maintains a sterile, nontoxic intraocular environment, limiting further damage to the retina and uveal tract. We recommend CEVE as the primary treatment for all fundus obscuring APCE ( $\sim 75 \%$ of cases), which includes all LP/HM eyes and approximately half of eyes with $\mathrm{CF}$ or better visual acuity. We reserve TAP for CF or better eyes in which the fundus view is adequate to rule out macular distress.

In cases where CEVE is planned but will be delayed, immediate in-office tap/inject can be considered. Regardless of initial therapy, clarity of the intraocular media should be closely followed with a low threshold to retreat with lack of improvement. In the era of small gauge vitrectomy, we believe the CEVE/CEVE+ paradigm provides the best chance of promptly halting and reversing this potentially devastating complication of cataract surgery.

\section{Ethics Statement}

This retrospective study was approved by the Western Institutional Review Board and adhered to the tenets of the Declaration of Helsinki for research involving human subjects.

\section{Acknowledgments}

We gratefully acknowledge the following: Jessica Haynes and Christina Sullivan for their extensive work in compiling data, images, and videos for this article; and the following fellowship physicians who helped care for the patients included in this study: Anthony Correnti, MD, Jeffrey L. Shere, MD, Brett D. Gerwin, MD, Alexander V. Talalight, MD, Wright B. Lauten, MD, Andrew D. Hsia, MD, Eric M. Zavaleta, MD, Charles L. Clark, III, MD, Daniel K. Bennett, MD, Nicholas H. Tosi, MD, Vikram T. Saini, MD, Daniel T. Kasuga, MD. Partial funding was provided by the Helen Keller Foundation for Research and Education through a grant from the Pete Hanna Charitable Trust and donations from the Kent Companies, Midland, Texas.

\section{Author Contributions}

Kuhn and Morris developed the CEVE paradigm. ${ }^{12,16-18}$ Morris, Sapp and Oltmanns developed the CEVE+ concept and treated the patients in this study. Dib compiled and performed the first analysis of the data and authored the first manuscript draft. Morris and Dib revised the manuscript through subsequent drafts. Morris performed three vitrectomies shown in supplemental videos and figures. All authors made a significant contribution to the work reported, whether that is in the conception, study design, execution, acquisition of data, analysis and interpretation, or in all these areas; took part in drafting, revising or critically reviewing the article; gave final approval of the version to be published; have agreed on the journal to which the article has been submitted; and agree to be accountable for all aspects of the work.

\section{Disclosure}

The authors report no conflicts of interest in this work, financial or otherwise.

\section{References}

1. West ES, Behrens A, McDonnell PJ, Tielsch JM, Schein OD. The incidence of endophthalmitis after cataract surgery among the U.S. medicare population increased between 1994 and 2001. Ophthalmology. 2005;112 (8):1388-1394. doi:10.1016/j.ophtha.2005.02.028 
2. Taban M, Behrens A, Newcomb RL, et al. Acute endophthalmitis following cataract surgery. Arch Ophthalmol. 2005;123(5):613. doi:10.1001/archopht.123.5.613

3. Doft B. Results of the Endophthalmitis Vitrectomy Study. A randomized trial of immediate vitrectomy and of intravenous antibiotics for the treatment of postoperative bacterial endophthalmitis. Endophthalmitis Vitrectomy Study Group. Arch Ophthalmol. 1995;113(12):1479-1496.

4. Wisniewski SR, Hammer ME, Grizzard WS. An investigation of the hospital charges related to the treatment of endophthalmitis in the endophthalmitis vitrectomy study. Ophthalmology. 1997;104(5):739745. doi:10.10.16/S0161-6420(97(30239-5

5. Yannuzzi NA, Si N, Relhan N, et al. Endophthalmitis after clear corneal cataract surgery: outcomes over two decades. Am J Ophthalmol. 2017;174:155-159. doi:10.1016/j.ajo.2016.11.006

6. Pijl BJ, Theelen T, Tilanus MAD, Rentenaar R, Crama N. Acute endophthalmitis after cataract surgery: 250 consecutive cases treated at a tertiary referral center in the Netherlands. Am J Ophthalmol. 2010;149(3):482-487.e2. doi:10.1016/j.ajo.2009.09.021

7. Mason LB, Mason JOI, Friedman DA, Mason JOI. Postoperative bacterial endophthalmitis: tap/inject versus sutureless vitrectomy. Med Res Archives. 2017. Available from: https://journals.ke-i.org/ mra/article/view/999. Accessed, 2020.

8. Lalwani GA, Flynn HW, Scott IU, et al. Acute-onset endophthalmitis after clear corneal cataract surgery (1996-2005). Clinical features, causative organisms, and visual acuity outcomes. Ophthalmology. 2008;115(3):473-476. doi:10.1016/j.ophtha.2007.06.006

9. Kamalarajah S, Silvestri G, Sharma N, et al. Surveillance of endophthalmitis following cataract surgery in the UK. Eye (Lond). 2004;18(6):580-587. doi:10.1038/sj.eye.6700645

10. Gower EW, Keay LJ, Stare DE, et al. Characteristics of endophthalmitis after cataract surgery in the United States medicare population. Ophthalmol. 2015;122(8):1625-1632. doi:10.1016/j.ophtha.2015.04. 036

11. Combey de Lambert A. Baseline factors predictive of visual prognosis in acute postoperative bacterial endophthalmitis in patients undergoing cataract surgery. JAMA Ophthalmol. 2013;131(9):1159. doi:10.1001/jamaophthalmol.2013.4242

12. Kuhn F, Gini G. Ten years after ... are findings of the Endophthalmitis Vitrectomy Study still relevant today? Graefes Arch Clin Exp Ophthalmol. 2005;243:1197-1199. doi:10.1007/ s00417-005-9982-8

13. Clarke B, Williamson TH, Gini G, Gupta B. Management of bacterial postoperative endophthalmitis and the role of vitrectomy. Surv Ophthalmol. 2018;63(5):677-693. doi:10.1016/j.survophthal.2018. 02.003

14. Maguire JI. Postoperative endophthalmitis: optimal management and the role and timing of vitrectomy surgery. Eye (Lond). 2008;22 (10):1290-1300. doi:10.1038/eye.2008.51

15. Doft B. Endophthalmitis Vitrectomy Study. Manual of Operations. National Eye Institute.; 1990.

16. Kuhn F. Complete and Early Vitrectomy for Endophthalmitis (CEVE) as today's alternative to the Endophthalmitis Vitrectomy Study. In: Kirchhof B, Wong D, editors. Essentials in Ophthalmology, VitreoRetinal Surgery. Berlin, Heidelberg: Springer-Verlag;2005:53-68. doi:10.1007/978-3-540-33670-9_5

Clinical Ophthalmology

\section{Publish your work in this journal}

Clinical Ophthalmology is an international, peer-reviewed journal covering all subspecialties within ophthalmology. Key topics include: Optometry; Visual science; Pharmacology and drug therapy in eye diseases; Basic Sciences; Primary and Secondary eye care; Patient Safety and Quality of Care Improvements. This journal is indexed on PubMed
17. Morris R, Witherspoon CD, Kuhn F, Byrne JB. Endophthalmitis. In: FH R, editor. Master Tech Ophthalmic Surgery. Philadelphia: Jaypee Brothers Medical Publishers; 1995:560-572.

18. Morris RE, Clark CL, Sapp MR, Oltmanns MH, Kuhn F. Endophthalmitis. In: Roy FH, editor. Master Tech Ophthalmic Surgery. 2nd ed. Philadelphia: Jaypee Brothers Medical Publishers; 2015:473-484.

19. Brockhaus L, Goldblum D, Eggenschwiler L, et al. Revisiting systemic treatment of bacterial endophthalmitis: a review of intravitreal penetration of systemic antibiotics. Clin Microbiol Infect. 2019;25 (11):1364-1369. doi:10.1016/j.cmi.2019.01.017

20. Plugfelder SC, Hernández E, Fliesler SJ, et al. Intravitreal vancomycin: retinal toxicity, clearance, and interaction with gentamicin. Arch Ophthalmol. 1987;105(6):831-837. doi:10.1001/archopht.1987.01060 060117045

21. Fliney GD, Pecen PE, Cathcart JN, Palestine AG. Trends in treatment strategies for suspected bacterial endophthalmitis. Graefes Arch Clin Exp Ophthalmol. 2018;256(4):833-838. doi:10.1007/s00417-018-3910-3

22. Callegan MC, Booth MC, Jett BD, Gilmore MS, Tuomanen EI. Pathogenesis of gram-positive bacterial endophthalmitis. Infect Immun. 1999;67(7):3348-3356.

23. Kim IT, Park SK, Lim JH. Inflammatory response in experimental Staphylococcus and Pseudomonas endophthalmitis. Ophthalmologica. 1999;213(5):305-310. doi:10.1159/000027444

24. Forster RK. Experimental postoperative endophthalmitis. Trans Am Ophthalmol Soc. 1992;90:505-559. doi:10.1001/archinte.1934.0016011 0018002

25. Callegan MC, Guess S, Wheatley NR, et al. Efficacy of vitrectomy in improving the outcome of Bacillus cereus endophthalmitis. Retina. 2011;31(8):1518-1524. doi:10.1097/IAE.0b013e318206d176

26. Zhou T, Aptel F, Bron AM, et al. Longitudinal study of retinal status using optical coherence tomography after acute onset endophthalmitis following cataract surgery. Br J Ophthalmol. 2017;101(9):12111216. doi:10.1136/bjophthalmol-2016-309542

27. Olson JC, Flynn HW, Forster RK, Culbertson WW. Results in the treatment of postoperative endophthalmitis. Ophthalmology. 1983;90 (6):692-699. doi:10.1016/S0161-6420(83)34511-5

28. Nelsen PT, Marcus DA, Bovino JA. Retinal detachment following endophthalmitis. Ophthalmology. 1985;92(8):1112-1117. doi:10.1016/ S0161-6420(85)33916-7

29. Chiquet C, Aptel F, Combey-de Lambert A, et al. Occurrence and risk factors for retinal detachment after pars plana vitrectomy in acute postcataract bacterial endophthalmitis. $\mathrm{Br} J$ Ophthalmol. 2016;100 (10):1388-1392. doi:10.1136/bjophthalmol-2015-307359

30. Doft BM, Kelsey SF, Wisniewski SR, et al. Retinal detachment in the endophthalmitis vitrectomy study. Arch Ophthalmol. 2000;118 (12):1661. doi:10.1001/archopht.118.12.1661

31. Doft BH, Kelsey SF, Wisniewski SR. Additional procedures after the initial vitrectomy or tap-biopsy in the endophthalmitis vitrectomy study. Ophthalmology. 1998;105(4):707-716. doi:10.1016/S01616420(98)94028-3

32. Brouwer MC, Coutinho JM, Van De Beek D. Clinical characteristics and outcome of brain abscess: systematic review and meta-analysis. Neurology. 2014;82(9):806-813. doi:10.1212/WNL.0000000000000172

\section{Dovepress}

Central and CAS, and is the official journal of The Society of Clinical Ophthalmology (SCO). The manuscript management system is completely online and includes a very quick and fair peer-review system, which is all easy to use. Visit http://www.dovepress.com/ testimonials.php to read real quotes from published authors. 